\title{
Fixed point theorems for precomplete numberings
}

\author{
Henk Barendregt ${ }^{\mathrm{a}}$, Sebastiaan A. Terwijn ${ }^{\mathrm{b}, *}$ \\ a Radboud University Nijmegen, Institute for Computing and Information Sciences, P.O. Box 9010, \\ 6500 GL Nijmegen, the Netherlands \\ b Radboud University Nijmegen, Department of Mathematics, P.O. Box 9010, 6500 GL Nijmegen, \\ the Netherlands
}

\section{A R T I C L E I N F O}

Article history:

Received 22 October 2018

Accepted 12 April 2019

Available online $\mathrm{xxxx}$

\section{$M S C$}

$03 \mathrm{D} 25$

03B 40

$03 \mathrm{D} 45$

03D80

Keywords:

Precomplete numberings

Ershov recursion theorem

ADN theorem

Arslanov completeness criterion

\begin{abstract}
A B S T R A C T
In the context of his theory of numberings, Ershov showed that Kleene's recursion theorem holds for any precomplete numbering. We discuss various generalizations of this result. Among other things, we show that Arslanov's completeness criterion also holds for every precomplete numbering, and we discuss the relation with Visser's ADN theorem, as well as the uniformity or nonuniformity of the various fixed point theorems. Finally, we base numberings on partial combinatory algebras and prove a generalization of Ershov's theorem in this context.
\end{abstract}

() 2019 Elsevier B.V. All rights reserved.

\section{Introduction}

In this paper we discuss various fixed point theorems in computability theory, and related areas such as $\lambda$-calculus and combinatory algebra. The starting point is Kleene's famous recursion theorem [15], which was generalized to precomplete numberings by Ershov [10]. These are discussed in section 3, after we first discuss Ershov's theory of numberings in section 2.

The recursion theorem was generalized in other ways by Visser [30] and Arslanov [2]. Visser proved the so-called 'anti diagonal normalization theorem' that we discuss in section 4. Arslanov extended the recursion theorem from computable functions to arbitrary functions computable from an incomplete c.e. Turing degree. The Arslanov completeness criterion states that a c.e. set is Turing complete if and only if it computes a fixed point free function. Recently, a joint generalization of Arslanov's completeness criterion and the ADN theorem was given by Terwijn [27]. We discuss Arslanov's completeness criterion in section 5.

\footnotetext{
* Corresponding author.

E-mail addresses: henk@cs.ru.nl (H. Barendregt), terwijn@math.ru.nl (S.A. Terwijn).
} 
Finally, in sections 6 and 7, we discuss the relation with Feferman's version of the recursion theorem for partial combinatory algebras (pca's) [11]. Here we base the notion of numbering on pca's of arbitrary cardinality, and prove a fixed point theorem for these (Theorem 6.5). This generalizes Ershov's recursion theorem in this setting.

Our notation from computability theory is mostly standard. In the following, $\varphi_{n}$ denotes the $n$-th partial computable (p.c.) function, in some standard numbering of the p.c. functions. Partial computable (p.c.) functions are denoted by lower case Greek letters, and (total) computable functions by lower case Roman letters. $\omega$ denotes the natural numbers. $W_{e}$ denotes the domain of the p.c. function $\varphi_{e}$. We write $\varphi_{e}(n) \downarrow$ if this computation is defined, and $\varphi_{e}(n) \uparrow$ otherwise. We let $\langle e, n\rangle$ denote a computable pairing function. $\emptyset^{\prime}$ denotes the halting set. For unexplained notions we refer to Odifreddi [21] or Soare [26].

\section{Numberings and equivalence relations}

The theory of numberings (also called numerations, after the German 'Numerierung') was initiated by Ershov. The following concepts were introduced by him in [8].

Definition 2.1. A numbering of a set $S$ is a surjection $\gamma: \omega \rightarrow S$. Given $\gamma$, define an equivalence relation on $\omega$ by $n \sim_{\gamma} m$ if $\gamma(n)=\gamma(m)$.

A numbering $\gamma$ is precomplete if for every partial computable unary function $\psi$ there exists a computable unary $f$ such that for every $n$

$$
\psi(n) \downarrow \Longrightarrow f(n) \sim_{\gamma} \psi(n) .
$$

Following Visser, we say that $f$ totalizes $\psi$ modulo $\sim_{\gamma}$ if $(1)$ holds.

A precomplete numbering $\gamma$ is complete if there is a special element $a \in \omega$ such that next to (1) also $f(n) \sim_{\gamma} a$ for every $n$ with $\psi(n) \uparrow$.

The prime example of a numbering is $n \mapsto \varphi_{n}$ for the set of unary p.c. functions. This numbering is precomplete: by the S-m-n-theorem, for any p.c. $\psi$ there is a (total) computable $f$ such that $\varphi_{f(n)}=\varphi_{\psi(n)}$ for every $n$ such that $\psi(n) \downarrow$. The numbering is even complete: as required special element we can take an index of the totally undefined function.

The numbering $n \mapsto W_{n}$ of the c.e. sets is closely related (and for our purposes below equivalent) to the numbering of the p.c. functions. It is also complete, with as special element the empty set.

Other examples of numberings come from $\lambda$-calculus. For example, the closed $\lambda$-terms, modulo $\beta$-equality, can be enumerated as a precomplete numbering, ${ }^{1}$ cf. Visser [30, p261,264], referring to Barendregt. If moreover unsolvable $\lambda$-terms are equated, then this numbering even becomes complete. Other examples can be found in [30], and still more examples come from pca's, that we discuss in section 6 below.

Numberings and equivalence relations are mutually related [6]. For every numbering $\gamma$ we have the corresponding equivalence $\sim_{\gamma}$. Conversely, given an equivalence relation $R$ on $\omega$ (or any other countable set), we have the numbering $n \mapsto[n]$ of the equivalence classes of $R$. Hence the study of numberings is equivalent to that of (countable) equivalence relations. In particular we can also apply the terminology of Definition 2.1 to such relations, and talk about precomplete and complete equivalence relations.

A class of countable equivalence relations that is of particular interest is the class of computably enumerable equivalence relations, simply called ceers. These were studied by Ershov [9] in the context of the theory of numberings (though examples of them occurred earlier in the literature), and in early writings

$\overline{1}$ By $\gamma(n)=E \mathbf{c}_{n}$, where $\mathrm{E}$ is a $\lambda$-term enumerating closed terms and $\mathbf{c}_{n}$ is the $n$-th numeral adequately representing natural numbers in $\lambda$-calculus. 
were called positive equivalence relations. Bernardi and Sorbi [6] proved that every precomplete ceer is m-complete (even with an extra uniformity condition). They also showed that this implies 1-completeness [6, p532]. This result was later strengthened by Lachlan [18] (see also [1, p425]), who showed that all precomplete ceers are computably isomorphic. For a recent survey about ceers we refer the reader to Andrews, Badaev, and Sorbi [1].

An interesting example of a ceer (discussed in Bernardi and Sorbi [6, p534]) is the Lindenbaum algebra of PA (Peano arithmetic). Identify formulas $\varphi$ and $\psi$ in the language of PA with their Gödel numbers. Let $\varphi \sim_{\mathrm{PA}} \psi$ if these formulas are provably equivalent in PA. Then $\sim_{\mathrm{PA}}$ is obviously a ceer. This relation is not precomplete, as can be seen using Theorem 3.1 below: the function $\varphi \mapsto \neg \varphi$ is computable, but does not have a fixed point modulo $\sim_{\mathrm{PA}}$. By contrast, the analogous ceer $\sim_{\Sigma_{n}}$, obtained by considering the fragment of PA of $\Sigma_{n}$-formulas, is precomplete, cf. Visser [30, p263].

\section{The recursion theorem}

Kleene's recursion theorem [15] states that every computable function $h$ has a fixed point, in the sense that there exists a number $n$ such that $\varphi_{h(n)}=\varphi_{n}$. This result holds uniformly, meaning that the fixed point can be found computably from a code of $h$. For an extensive discussion of this fundamental theorem, and the many applications it has found in logic, see Moschovakis [20].

Using Ershov's terminology, we can phrase Kleene's result by saying that $h$ has a fixed point modulo $\sim_{\gamma}$, where $\gamma$ is the numbering $n \mapsto \varphi_{n}$ of the p.c. functions. Ershov showed that the recursion theorem holds for every precomplete numbering $\gamma$ in the following way.

Theorem 3.1. (Ershov's recursion theorem [10]) Let $\gamma$ be a precomplete numbering, and let $h$ be a computable function. Then $h$ has a fixed point modulo $\sim_{\gamma}$, i.e. there exists a number $n$ such that $h(n) \sim_{\gamma} n$.

As is the case for Kleene's recursion theorem, this result holds uniformly. For later reference we explicitly state the following version:

Theorem 3.2. (Ershov's recursion theorem with parameters) Let $\gamma$ be a precomplete numbering, and let $h(x, n)$ be a computable binary function. Then there exists a computable function $f$ such that for all $n$, $f(n) \sim_{\gamma} h(f(n), n)$.

Proof. By precompleteness, let $d$ be a computable function such that

$$
d(x, n) \sim_{\gamma} \varphi_{x}(x, n)
$$

for every $x$ and $n$ where the latter is defined. ${ }^{2}$ Let $e$ be a code such that $\varphi_{e}(x, n)=h(d(x, n), n)$ for all $x$ and $n$. Then

$$
d(e, n) \sim_{\gamma} \varphi_{e}(e, n)=h(d(e, n), n)
$$

so that $d(e, n)$ is a fixed point for every $n$.

Theorem 3.2 is equivalent with the following form, given in Andrews, Badaev, and Sorbi [1, p423].

\footnotetext{
2 Note that we can generalize precompleteness (Definition 2.1) to functions with multiple arguments, which is allowed by the usual coding of sequences.
} 
Theorem 3.3. Let $\gamma$ be a precomplete numbering. There exists a computable function $f$ such that for every $n$, if $\varphi_{n}(f(n)) \downarrow$ then

$$
\varphi_{n}(f(n)) \sim_{\gamma} f(n) .
$$

Theorem 3.2 and Theorem 3.3 are equivalent, for precomplete numberings. To see that Theorem 3.3 implies Theorem 3.2, observe that, given a computable function $h$ as in the latter theorem, there is a computable function $g$ such that $\varphi_{g(n)}(x)=h(x, n)$ for every $x$ and $n$. For $f$ as in Theorem 3.3 we then have

$$
h(f(g(n)), n)=\varphi_{g(n)}(f(g(n))) \sim_{\gamma} f(g(n))
$$

for every $n$, so $f \circ g$ is the desired computable function producing fixed points.

Conversely, Theorem 3.2 implies Theorem 3.3. By precompleteness of $\gamma$, there is a computable function $h$ that totalizes the universal p.c. function modulo $\sim_{\gamma}$, i.e. such that

$$
\varphi_{n}(x) \downarrow \Longrightarrow h(x, n)=\varphi_{n}(x)
$$

for every $x$ and $n$. Now Theorem 3.2 provides the required fixed points $f(n)$.

The converse of Theorem 3.3 also holds. The statement of the theorem holds for a numbering $\gamma$ if and only if $\gamma$ is precomplete (cf. [1, p423]). Since the equivalence of Theorem 3.2 and Theorem 3.3 above uses that $\gamma$ is precomplete, it is not clear whether the converse of Theorem 3.2 also holds. Hence we ask the following.

Question 3.4. Suppose that an arbitrary numbering $\gamma$ satisfies the statement of Theorem 3.2. Does it follow that $\gamma$ is precomplete?

\section{The ADN theorem}

The ADN theorem (Theorem 4.2 below) is an extension of the recursion theorem, proved in Visser [30]. It was motivated by developments in early proof theory, in particular Rosser's extension of Gödel's incompleteness theorem. Visser mentions the work of Smoryński and Shepherdson's fixed point as further motivation, cf. [25]. The analogy between the ADN theorem and Rosser's theorem was neatly summarized in Barendregt [5] by the following mock equation.

$$
\frac{\text { Gödel }}{\text { Rosser }}=\frac{\text { recursion theorem }}{\text { ADN theorem }}
$$

The analogy is further illustrated by the proof of the ADN theorem below.

The ADN theorem has several interesting applications.

- Visser himself discusses some consequences of the ADN theorem for the $\lambda$-calculus in [30].

- Theorem 1 (about the m-completeness of precomplete ceers) in Bernardi and Sorbi [6] uses $\omega+1$ applications of the ADN theorem. The construction in the proof uses the ADN theorem $\omega$ times, plus one more for Lemma 2.

- Barendregt [5] uses the ADN theorem to prove a result of Statman.

- The notion of diagonal function used in the ADN theorem relates nicely to the concept of fixed point free function and similar concepts that figure prominently in computability theory, cf. the discussion below. 
Definition 4.1. A partial function $\delta$ is a diagonal function for the numbering $\gamma$ if $\delta(x) \nsim_{\gamma} x$ for every $x$ in the domain of $\delta$.

N.B. Note that in this definition we do not require $\delta$ to be p.c., in contrast to the original definition in Visser [30]. This is because it is also interesting to discuss the Turing degrees of diagonal functions in general.

By Jockusch et al. [12], the Turing degrees of diagonal functions for the numberings $n \mapsto \varphi_{n}$ and $n \mapsto W_{n}$ coincide. They also coincide with the degrees of diagonally noncomputable, or DNC, functions, i.e. functions $g$ with $g(e) \neq \varphi_{e}(e)$ for every $e$. Diagonal functions for the numbering $n \mapsto W_{n}$ of the c.e. sets are called fixed point free (or simply FPF) in the literature. (Usually these are total functions, though in [27] and [28] also partial FPF functions were considered.) DNC and FPF functions play an important part in computability theory, for example in the work of Kučera [17]. See Astor [3] for a recent example of their use, or Downey and Hirschfeldt [7] for many more. They are also closely related to the study of complete extensions of Peano Arithmetic, see e.g. the work of Jockusch and Soare [13].

Theorem 4.2. (ADN theorem, Visser [30]) Let $\gamma$ be a precomplete numbering, and suppose that $\delta$ is a partial computable diagonal function for $\gamma$. Then for every partial computable function $\psi$ there exists a computable function $f$ such that for every $n$,

$$
\begin{aligned}
\psi(n) \downarrow & \Longrightarrow f(n) \sim_{\gamma} \psi(n) \\
\psi(n) \uparrow & \Longrightarrow \delta(f(n)) \uparrow
\end{aligned}
$$

Definition 4.3. Note that (2) expresses that $f$ totalizes $\psi$ modulo $\sim_{\gamma}$. If both (2) and (3) hold, we say that $f$ totalizes $\psi$ avoiding $\delta$.

Note that the ADN theorem implies Ershov's recursion theorem (Theorem 3.1). Indeed, suppose towards a contradiction that some total computable $d$ has no fixed point modulo $\sim_{\gamma}$. Then $d$ is a total computable diagonal function. Then a p.c. function $\psi$ with $\psi(0) \uparrow$ cannot be totalized modulo $\sim_{\gamma}$ avoiding $d$ by any $f$, as we will not have $d(f(0)) \uparrow$, by the totality of $d$. This contradicts the ADN Theorem.

Proof of Theorem 4.2. We use Ershov's recursion theorem with parameters (Theorem 3.2). Let $\eta$ be p.c. such that for all $x$ and $n$,

$$
\eta(x, n)= \begin{cases}\delta(x) \quad \text { if } \delta(x) \downarrow<\psi(n) \downarrow \text { (by this we mean that the first stage at which } \delta(x) \text { converges } \\ \quad \text { is less than that of } \psi(n), \text { if the latter converges at all), } \\ \psi(n) \quad \text { if } \psi(n) \downarrow \leq \delta(x) \downarrow, \\ \uparrow \quad \text { otherwise. }\end{cases}
$$

By precompleteness of $\gamma$, there is a computable function $h$ that totalizes $\eta$ modulo $\sim_{\gamma}$. Let $f$ be as in Ershov's recursion theorem with parameters (Theorem 3.2). Then for every $n$,

$$
f(n) \sim_{\gamma} h(f(n), n) \sim_{\gamma} \eta(f(n), n),
$$

whenever the latter is defined. Now $\delta(f(n)) \downarrow$ and $f(n) \sim_{\gamma} \delta(f(n))$ is impossible, since $\delta$ is a diagonal for $\gamma$, and hence $f$ totalizes $\psi$ avoiding $\delta$.

By taking $\psi$ in Theorem 4.2 universal, we see that the following uniform version holds. 
Theorem 4.4. (ADN theorem, uniform version) Let $\gamma$ be a precomplete numbering, and suppose that $\delta$ is a partial computable diagonal function for $\gamma$. Then there exists a computable function $f$ such that for every fixed e the function $f(\langle e, n\rangle)$ totalizes $\varphi_{e}$ avoiding $\delta$.

Proof. Consider the universal function $\psi(\langle e, n\rangle)=\varphi_{e}(n)$. By Theorem 4.2, there exists a computable $f$ that totalizes $\psi$ avoiding $\delta$. Hence

$$
\begin{aligned}
& \psi(\langle e, n\rangle)=\varphi_{e}(n) \downarrow \Longrightarrow f(\langle e, n\rangle) \sim_{\gamma} \varphi_{e}(n) \\
& \psi(\langle e, n\rangle)=\varphi_{e}(n) \uparrow \Longrightarrow \delta(f(\langle e, n\rangle)) \uparrow,
\end{aligned}
$$

and therefore $f(\langle e, n\rangle)$ totalizes $\varphi_{e}$ avoiding $\delta$.

Theorem 4.4 shows that Theorem 4.2 is uniform in a code of $\psi$. A careful reading of the proof of the ADN theorem above shows that it is also uniform in a code $d$ of $\delta .{ }^{3}$ It is shown in Terwijn [28] that (for the numbering $n \mapsto W_{n}$ ) neither Arslanov's completeness criterion nor the ADN theorem have a version with parameters analogous to the recursion theorem with parameters. (Note that the ADN theorem is in a way a contrapositive formulation of the recursion theorem, so that some care is needed in how to formulate the parameterized version.) A fortiori, the same holds in the context of arbitrary precomplete numberings.

\section{Arslanov's completeness criterion for precomplete numberings}

Arslanov's completeness criterion [2] states that a c.e. set $A$ is Turing complete if and only if $A$ can compute a FPF function, i.e. a function $f$ such that $W_{f(n)} \neq W_{n}$ for every $n$. Note that this vastly extends Kleene's recursion theorem, namely from computable sets to incomplete c.e. sets. The condition that $A$ is c.e. is necessary, as by the low basis theorem [14] there exist FPF functions of low Turing degree.

In the next theorem we formulate Arslanov's completeness criterion for arbitrary precomplete numberings. The usual version of the completeness criterion corresponds to the case where $\gamma$ is the standard numbering of the c.e. sets $n \mapsto W_{n}$. (Or equivalently, by the aforementioned result of Jockusch et al. [12], the numbering of the p.c. functions $n \mapsto \varphi_{n}$.) Below, $\emptyset^{\prime}$ denotes the halting set, and $\emptyset_{s}^{\prime}$ denotes its $s$-step approximation.

Theorem 5.1. Suppose $\gamma$ is a precomplete numbering, and $A<_{T} \emptyset^{\prime}$ is an incomplete c.e. set. If $g$ is an A-computable function, then $g$ has a fixed point modulo $\gamma$, i.e. there exists $n \in \omega$ such that $g(n) \sim_{\gamma} n$.

Proof. The following proof is a modification of the proof in Soare [26].

Since $g \leq_{T} \emptyset^{\prime}$, by Shoenfield's limit lemma [24] there is a computable approximation $\hat{g}$ such that

$$
g(n)=\lim _{s \rightarrow \infty} \hat{g}(n, s)
$$

for every $n$. Because $A$ is c.e., this approximation has a modulus $m \leq_{T} A$, that is, for all $s \geq m(n)$ we have $g(n)=\hat{g}(n, s)$. Now let $\eta$ be partial computable such that

$$
\eta(x, n)= \begin{cases}\hat{g}\left(x, s_{n}\right) & \text { if } s_{n} \text { is the least number } s \text { such that } n \in \emptyset_{s}^{\prime}, \\ \uparrow & \text { if such } s \text { does not exist. }\end{cases}
$$

By the precompleteness of $\gamma$, let $h$ be a computable function that totalizes $\eta$ modulo $\sim_{\gamma}$, so that $h(x, n) \sim_{\gamma}$ $\eta(x, n)$ whenever the latter is defined. By Ershov's recursion theorem with parameters (Theorem 3.2), let

3 This means that there is a computable function $f=f(d, n)$ such that, if $\delta=\varphi_{d}$ is a diagonal function for $\gamma$, then $f(d, n)$ totalizes $\psi$ avoiding $\delta$. 
$f$ be a computable function such that $f(n) \sim_{\gamma} h(f(n), n)$ for every $n$. In particular we have $f(n) \sim_{\gamma}$ $\eta(f(n), n) \sim_{\gamma} \hat{g}\left(f(n), s_{n}\right)$ when $n \in \emptyset^{\prime}$.

We claim that there exists $n \in \emptyset^{\prime}$ such that $\hat{g}\left(f(n), s_{n}\right)=g(f(n))$, so that $f(n)$ is a fixed point of $g$. Otherwise we would have that for every $n$, if $n \in \emptyset^{\prime}$ then $\hat{g}\left(f(n), s_{n}\right) \neq g(f(n))$, and hence $m(f(n))>s_{n}$. It follows that $n \in \emptyset^{\prime} \Leftrightarrow n \in \emptyset_{m(f(n))}^{\prime}$, and hence $\emptyset^{\prime} \leq_{T} A$, contrary to assumption.

A joint generalization of the ADN theorem and Arslanov's completeness criterion for the numbering $n \mapsto W_{n}$ of the c.e. sets was given in Terwijn [27]. At this point it is not clear that the proof in [27] generalizes to arbitrary precomplete numberings, though we conjecture that it is possible to adapt the proof.

Question 5.2. Does the joint generalization Theorem 5.1 in [27] hold for arbitrary precomplete numberings?

\section{Numberings and partial combinatory algebra}

In this section we discuss the relation of the theory of numberings with partial combinatory algebra. Combinatory algebra was introduced by Schönfinkel [23], and partial combinatory algebra in Feferman [11]. We begin by repeating some relevant definitions. A fuller account of partial combinatory algebra can be found in van Oosten [22], from which we also borrow some of the terminology.

A partial applicative structure (pas) is a set $\mathcal{A}$ together with a partial map from $\mathcal{A} \times \mathcal{A}$ to $\mathcal{A}$. We denote the image of $(a, b)$, if it is defined, by $a b$, and think of this as ' $a$ applied to $b$ '. If this is defined we denote this by $a b \downarrow$. By convention, application associates to the left. We write $a b c$ instead of $(a b) c$. Terms over $\mathcal{A}$ are built from elements of $\mathcal{A}$, variables, and application. If $t_{1}$ and $t_{2}$ are terms then so is $t_{1} t_{2}$. If $t\left(x_{1}, \ldots, x_{n}\right)$ is a term with variables $x_{i}$, and $a_{1}, \ldots, a_{n} \in \mathcal{A}$, then $t\left(a_{1}, \ldots, a_{n}\right)$ is the term obtained by substituting the $a_{i}$ for the $x_{i}$. For closed terms (i.e. terms without variables) $t$ and $s$, we write $t \simeq s$ if either both are undefined, or both are defined and equal. Here application is strict in the sense that for $t_{1} t_{2}$ to be defined, it is necessary (but not sufficient) that both $t_{1}, t_{2}$ are defined. We say that an element $f \in \mathcal{A}$ is total if $f a \downarrow$ for every $a \in \mathcal{A}$.

Definition 6.1. A partial applicative structure $\mathcal{A}$ is combinatory complete if for any term $t\left(x_{1}, \ldots, x_{n}, x\right)$, with free variables among $x_{1}, \ldots, x_{n}, x$ and $0 \leq n$, there exists a $b \in \mathcal{A}$ such that for all $a_{1}, \ldots, a_{n}, a \in \mathcal{A}$,

(i) $b a_{1} \cdots a_{n} \downarrow$,

(ii) $b a_{1} \cdots a_{n} a \simeq t\left(a_{1}, \ldots, a_{n}, a\right)$.

A pas $\mathcal{A}$ is a partial combinatory algebra (pca) if it is combinatory complete.

The property of combinatory completeness allows for the following definition in any pca. ${ }^{4}$ For every term $t\left(x_{1}, \ldots, x_{n}, x\right), 0 \leq n$, with free variables among $x_{1}, \ldots, x_{n}, x$, one can explicitly define a term $\lambda^{*} x . t$ with variables among $x_{1}, \ldots, x_{n}$, with the property that for all $a_{1}, \ldots, a_{n}, a \in \mathcal{A}$,

(i) $\left(\lambda^{*} x . t\right)\left(a_{1}, \ldots, a_{n}\right) \downarrow$,

(ii) $\left(\lambda^{*} x . t\right)\left(a_{1}, \ldots, a_{n}\right) a \simeq t\left(a_{1}, \ldots, a_{n}, a\right)$.

This is noted in Feferman [11, p95], and makes use of the Curry combinators $k$ and $s$ familiar from combinatory logic. In fact, for any pas, the existence of such combinators is equivalent to being a pca [22, p3].

\footnotetext{
4 Because the lambda-terms in combinatory algebra do not have the same substitution properties as in the lambda calculus, we use the notation $\lambda^{*}$ rather than $\lambda$.
} 
The prime example of a pca is Kleene's first model $\mathcal{K}_{1}$, consisting of $\omega$, with application defined by $n m=\varphi_{n}(m)$. This structure is combinatory complete by the S-m-n-theorem. However, there are many other examples, including uncountable structures, see Section 1.4 of [22].

Another important example of a pca is Kleene's second model $\mathcal{K}_{2}$ [16]. This is a pca defined on Baire space $\omega^{\omega}$ (often informally referred to as the 'reals'), with application defined by coding partial continuous functionals by reals. The application $\alpha \beta$ is then the result of applying the functional with code $\alpha$ to the real $\beta$. We will use this model below in section 7 . It also plays an important role in the theory of realizability and higher-order computability. For a more elaborate discussion see for example Longley and Normann [19].

The structures $\mathcal{K}_{1}$ and $\mathcal{K}_{2}$ can also be considered as total combinatory algebras if one restricts them to combinators corresponding to $\lambda \mathrm{I}$-calculus, in which the formation of $\lambda x . M$ only is allowed if $x$ is a free variable of $M$, see Barendregt [4, Exercises 9.5.13-14].

We note the following about the pca $\mathcal{K}_{1}$.

- The notion of precompleteness (1) generalizes the property that one can totalize any p.c. function $\psi$ on codes. This property gives Ershov's form of the recursion theorem (Theorem 3.1).

- Pca's generalize the applicative structure of $n m=\varphi_{n}(m)$. The property of combinatory completeness may be seen as an abstraction of Kleene's S-m-n-theorem. This property also gives rise to a fixed point theorem (due to Feferman), see Theorem 6.2 below.

These two generalizations of properties of Kleene's model are more or less orthogonal. For numberings, there is no notion of application, and pca's need not be countable.

The following is Feferman's form of the recursion theorem in pca's, inspired by the fixed point theorem in combinatory logic.

Theorem 6.2. (Feferman's recursion theorem [11]) Let $\mathcal{A}$ be a pca. Then there exists $f \in \mathcal{A}$ such that for all $g \in \mathcal{A}$

$$
g(f g) \simeq f g .
$$

Comparing this to Ershov's recursion theorem (in the form of Theorem 3.3), we see that Feferman's version is more general in that it applies to arbitrary pca's, but that it is also weaker in that the 'function' $f$ giving the fixed point $f g$ does not have to be total. ${ }^{5}$ In some cases $f$ may be total (as for example in Theorem 3.3, or in the case that $\mathcal{A}$ is a combinatory algebra, i.e. a pca in which application is total), but in general $f$ cannot be total. This is obviously the case when the pca has a totally undefined element $g .{ }^{6}$ We will comment further on this at the end of section 7 .

We now proceed by showing how a combination of the fixed point theorems of Ershov and Feferman can be obtained. We extend the notions of numbering and precompleteness of numberings from $\omega$ to arbitrary pca's as follows.

Definition 6.3. Suppose that $\mathcal{A}$ is a pca, $S$ is a set, and $\gamma: \mathcal{A} \rightarrow S$ is surjective. We call $\gamma$ a (generalized) numbering. Define an equivalence relation on $\mathcal{A}$ by $a \sim_{\gamma} b$ if $\gamma(a)=\gamma(b)$.

\footnotetext{
5 There is a second version of the recursion theorem for pca's in [22], namely that there is a term $f \in \mathcal{A}$ such that $f g \downarrow$ for every $g$, and such that $g(f g) a \simeq f g a$ for every $a \in \mathcal{A}$. Since $f$ is total, this version does imply Theorem 3.3 , but only for the special case of the numbering $n \mapsto \varphi_{n}$.

${ }^{6}$ This is in fact the case in every nontotal combinatory algebra. As soon as there is one application $a b$ that is undefined, $\mathcal{A}$ has a totally undefined element, namely $f=\lambda^{*} x \cdot a b=s(k a)(k b)$. In this case $f$ clearly satisfies Theorem 6.2. Although the theorem is thus quite weak as an extension from combinatory algebra, its use for us is that it suggests the generalization of Ershov's recursion theorem to pca's that we prove below (Theorem 6.5).

An alternative formulation of the recursion theorem in pca's, analogous to Theorem 3.3, would be: There exists a total $f \in \mathcal{A}$ such that for all $g \in \mathcal{A}$, if $g(f g) \downarrow$ then $g(f g) \sim f g$. This, however, does not hold in general by Proposition 7.3 below.
} 
Call $\gamma$ precomplete if for every term $t(x)$ with one variable $x$, there exists a total element $f \in \mathcal{A}$ such that

$$
t(a) \downarrow \Longrightarrow f a \sim_{\gamma} t(a)
$$

for every $a \in \mathcal{A}$. In this case, we say that $f$ totalizes $t$ modulo $\sim_{\gamma}$.

As before, we say that a generalized precomplete numbering $\gamma$ is complete if there is a special element $c \in \mathcal{A}$ such that in addition to (4), $f a \sim_{\gamma} c$ for every $a$ with $t(a) \uparrow$.

Lemma 6.4. Let $\mathcal{A}$ be a pca, and let $\gamma: \mathcal{A} \rightarrow S$ be a generalized numbering. Then the following are equivalent.

(i) $\gamma$ is precomplete.

(ii) For every $b \in \mathcal{A}$ there exists a total element $f \in \mathcal{A}$ such that for all $a \in \mathcal{A}$,

$$
b a \downarrow \Longrightarrow f a \sim_{\gamma} b a .
$$

(iii) For every $b \in \mathcal{A}$ there exists a total element $f \in \mathcal{A}$ such that for all $n \in \omega$ and $\vec{a}=a_{1}, \ldots, a_{n} \in \mathcal{A}$,

$$
b \vec{a} \downarrow \Longrightarrow f \vec{a} \sim_{\gamma} b \vec{a} .
$$

Proof. (i) $\Rightarrow$ (ii). Apply (i) to the term $b x$.

(ii) $\Rightarrow$ (iii). With the use of the $\lambda^{*}$-terms defined above for any pca, $n$-tuples $a_{1}, \ldots, a_{n}$ can be coded as a single element $\left\langle a_{1}, \ldots, a_{n}\right\rangle=\lambda^{*} z . z a_{1} \ldots a_{k}$, from which each $a_{i}$ can be decoded. Indeed, for $\mathbf{U}_{i}^{n}=$ $\lambda^{*} u_{1} \ldots u_{n} . u_{i}$ we have

$$
\left\langle a_{1}, \ldots, a_{n}\right\rangle \mathbf{U}_{i}^{n}=a_{i} .
$$

Now given $n, b$ define $b^{\prime}=\lambda^{*} z \cdot b\left(z \mathrm{U}_{1}^{n}\right) \cdots\left(z \mathrm{U}_{n}^{n}\right)$. Let $f^{\prime}$ totalize $b^{\prime}$ modulo $\gamma$. Then $f=\lambda^{*} x_{1} \ldots x_{n} \cdot f^{\prime}\left\langle x_{1}, \ldots\right.$, $\left.x_{n}\right\rangle$ totalizes $b$ : if $b a_{1} \cdots a_{n} \downarrow$, then

$$
\begin{aligned}
f a_{1} \cdots a_{n} & =f^{\prime}\left\langle a_{1}, \ldots, a_{n}\right\rangle \\
& \sim_{\gamma} b^{\prime}\left\langle a_{1}, \ldots, a_{n}\right\rangle \\
& =b\left(\left\langle a_{1}, \ldots, a_{n}\right\rangle \bigcup_{1}^{n}\right) \cdots\left(\left\langle a_{1}, \ldots, a_{n}\right\rangle \cup_{n}^{n}\right) \\
& =b a_{1} \cdots a_{n} .
\end{aligned}
$$

(iii) $\Rightarrow$ (i). Given term $t(x)$, apply (iii) with $n=1$ to $b=\lambda^{*} x \cdot t(x)$.

By Lemma 6.4, the notion of precompleteness from Definition 2.1 is a special case of Definition 6.3, namely the case where $\mathcal{A}$ is the pca $\mathcal{K}_{1}$, with application $n m=\varphi_{n}(m)$. Hence we see that Ershov's recursion theorem (Theorem 3.3) is a special case of the following theorem.

Theorem 6.5. Suppose $\mathcal{A}$ is a pca, and that $\gamma: \mathcal{A} \rightarrow S$ is a precomplete numbering. Then there exists a total $f \in \mathcal{A}$ such that for all $g \in \mathcal{A}$, if $g(f g) \downarrow$ then

$$
g(f g) \sim_{\gamma} f g
$$

Proof. The proof mimics $\Theta=\left(\lambda^{*} x y \cdot y(x x y)\right)\left(\lambda^{*} x y \cdot y(x x y)\right)$, the fixed point operator of Turing [29]. Let $t(x, y)=y(x x y)$. By Lemma 6.4 there is a $u \in \mathcal{A}$ that totalizes the term $t(x, y)$ modulo $\sim_{\gamma}$. Then $u a b \downarrow$, for all $a, b \in \mathcal{A}$, and $b(a a b) \downarrow$ implies $u a b \sim_{\gamma} b(a a b)$. Take $f=u u$. Then $f$ is total, because $u u a \downarrow$ for every $a \in \mathcal{A}$. Suppose for a $g \in \mathcal{A}$ one has $g(f g) \downarrow$. Then $g(u u g) \downarrow$ and 


$$
\begin{aligned}
f g & =u u g \\
& \sim_{\gamma} g(u u g) \\
& =g(f g) .
\end{aligned}
$$

In order to combine the fixed point theorems of Ershov and Feferman, we have extended the definition of numberings to pca's. It could also be interesting to investigate how much of the theory can be expressed in terms of realizability toposes derived from the pca's, cf. [22].

\section{Combinatory completeness and precompleteness}

With every pca $\mathcal{A}$ we have an associated generalized numbering $\gamma_{\mathcal{A}}: \mathcal{A} \rightarrow \mathcal{A}$, which is just the identity. We will discuss the relation between combinatory completeness of $\mathcal{A}$ and the precompleteness of $\gamma_{\mathcal{A}}$.

Combinatory completeness is the property in pca's analogous to the S-m-n-theorem, and precompleteness of (generalized) numberings (Definition 6.3) generalizes the property that every p.c. function can be totalized modulo equivalence of codes, i.e. that the numbering $n \mapsto \varphi_{n}$ is precomplete. Now the latter fact is proved using the S-m-n-theorem, so one might think that perhaps the property of combinatory completeness of a pca $\mathcal{A}$ implies that of precompleteness of the associated numbering $\gamma_{\mathcal{A}}$. We now show that this is not the case, and hence that the assumption of precompleteness in Theorem 6.5 is not superfluous. Recall Kleene's second model $\mathcal{K}_{2}$ from section 6 .

Proposition 7.1. Kleene's second model $\mathcal{K}_{2}$ is not precomplete, meaning that its associated generalized numbering $\gamma_{\mathcal{K}_{2}}$ is not precomplete.

Proof. According to Lemma 6.4, we have to prove that there is a partial continuous functional $\psi: \omega^{\omega} \rightarrow \omega^{\omega}$ that does not have a total continuous extension. For every finite string $\sigma \in \omega^{<\omega}$, denote by $[\sigma]$ the basic open set consisting of all $X \in \omega^{\omega}$ that have $\sigma$ as an initial segment. Now define $\psi$ on every basic open $\left[0^{n} 1\right]$ by mapping it continuously to $\left[0^{n} 1\right]$ if $n$ is even, and to $\left[10^{n-1}\right]$ if $n$ is odd. We let $\psi$ be undefined on the rest of $\omega^{\omega}$. Then $\psi$ is continuous on its domain. Now consider the all zero sequence $0^{\omega}$, and suppose that $f$ is a total continuous extension of $\psi$. Since the reals $0^{n} 10^{\omega}$ converge to $0^{\omega}$ for $n \rightarrow \infty$, their images under $f$ should converge to $f\left(0^{\omega}\right)$. But for even $n, f\left(0^{n} 10^{\omega}\right)$ tends to $0^{\omega}$, and for odd $n$ it tends to $10^{\omega}$. Hence every continuous extension $f$ of $\psi$ must have both $f\left(0^{\omega}\right)=0^{\omega}$ and $f\left(0^{\omega}\right)=10^{\omega}$, which is impossible.

Corollary 7.2. Combinatory completeness of a pca $\mathcal{A}$ does not imply precompleteness of the associated numbering $\gamma_{\mathcal{A}}$.

Proof. As $\mathcal{K}_{2}$ is a pca, this is immediate from Proposition 7.1.

We already noted that in general it is not possible to have the $f$ in Feferman's recursion theorem (Theorem 6.2) total. For $\mathcal{K}_{2}$, we can in fact say a bit more.

Proposition 7.3. In Kleene's second model $\mathcal{K}_{2}$, for every total element $f$ there exists a total element $g$ such that $g(f g) \not f f g$.

Proof. Given the code $f$ of a total continuous functional on $\omega^{\omega}$, we define a total continuous functional $g$ such that $g(f g) \neq f g$.

The particulars of the coding of $\mathcal{K}_{2}$ are not essential to the proof. (The interested reader can find them in Longley and Normann [19].) What is needed is that if $f g \downarrow$, this computation uses only a finite part of the coding of $g$ (this is precisely what it means for $f$ to be continuous on its domain), and further that the 
code of an element $g$ can be equal to an initial part of the code of the totally undefined function, and later become defined on a given number. Informally, the strategy to define $g$ is then as follows. First let $g$ be totally undefined, until $f g$ commits to a certain value on $(f g)(0)$. This has to happen since $f$ is total. We can then diagonalize by letting the value $(g(f g))(0)$ be different from $(f g)(0)$, as well as make $g$ total.

Note that Proposition 7.3 gives another proof of Proposition 7.1. Namely, if $\mathcal{K}_{2}$ were precomplete, then by Theorem 6.5 there would be a total element $f$ producing the fixed points, contradicting Proposition 7.3.

\section{Conflict of interest statement}

Declarations of interest: none.

\section{Acknowledgement}

The second author thanks Jaap van Oosten for helpful discussions about partial combinatory algebra.

\section{References}

[1] U. Andrews, S. Badaev, A. Sorbi, A survey on universal computably enumerable equivalence relations, in: A. Day, et al. (Eds.), R. Downey Festschrift, in: Lecture Notes in Computer Science, vol. 10010, Springer, 2017, pp. $418-451$.

[2] M.M. Arslanov, On some generalizations of the fixed point theorem, Sov. Math. (Izvestiya VUZ. Matematika) 25 (5) (1981) 1-10 (English translation).

[3] E. Astor, The computational content of intrinsic density, J. Symbolic Logic 83 (2) (2018) 817-828.

[4] H.P. Barendregt, The Lambda Calculus, 2nd edition, Studies in Logic and the Foundations of Mathematics, vol. 103, North-Holland, Amsterdam, 1984.

[5] H.P. Barendregt, Representing 'undefined' in lambda calculus, J. Funct. Programming 2 (3) (1992) $367-374$.

[6] C. Bernardi, A. Sorbi, Classifying positive equivalence relations, J. Symbolic Logic 48 (3) (1983) $529-538$.

[7] R.G. Downey, D.R. Hirschfeldt, Algorithmic Randomness and Complexity, Springer-Verlag, 2010.

[8] Y.L. Ershov, Theorie der Numerierungen I, Z. Math. Log. Grundl. Math. 19 (1973) 289-388.

[9] Y.L. Ershov, Positive equivalences, Algebra Logic 10 (1973) 378-394 (English translation).

[10] Y.L. Ershov, Theorie der Numerierungen II, Z. Math. Log. Grundl. Math. 21 (1975) 473-584.

[11] S. Feferman, A language and axioms for explicit mathematics, in: J.N. Crossley (Ed.), Algebra and Logic, Springer, 1975, pp. $87-139$.

[12] C.G. Jockusch jr., M. Lerman, R.I. Soare, R.M. Solovay, Recursively enumerable sets modulo iterated jumps and extensions of Arslanov's completeness criterion, J. Symbolic Logic 54 (4) (1989) 1288-1323.

[13] C.G. Jockusch jr., R.I. Soare, Degrees of members of $\Pi_{1}^{0}$ classes, Pacific J. Math. 40 (1972) 605-616.

[14] C.G. Jockusch jr., R.I. Soare, $\Pi_{1}^{0}$ classes and degrees of theories, Trans. Amer. Math. Soc. 173 (1972) $33-56$.

[15] S.C. Kleene, On notation for ordinal numbers, J. Symbolic Logic 3 (1938) 150-155.

[16] S.C. Kleene, R.E. Vesley, The Foundations of Intuitionistic Mathematics, North-Holland, 1965.

[17] A. Kučera, An Alternative, Priority-Free Solution to Post's Problem, Lecture Notes in Computer Science, vol. 233, Springer, 1986, pp. 493-500.

[18] A.H. Lachlan, A note on positive equivalence relations, Z. Math. Log. Grundl. Math. 33 (1987) $43-46$.

[19] J. Longley, D. Normann, Higher-Order Computability, Springer, 2015.

[20] Y.N. Moschovakis, Kleene's amazing second recursion theorem, Bull. Symbolic Logic 16 (2) (2010) 189-239.

[21] P. Odifreddi, Classical Recursion Theory, vol. 1, Studies in Logic and the Foundations of Mathematics, vol. 125, NorthHolland, Amsterdam, 1989.

[22] J. van Oosten, Realizability: An Introduction to Its Categorical Side, Studies in Logic and the Foundations of Mathematics, vol. 152, Elsevier, 2008.

[23] M. Schönfinkel, Über die Bausteine der mathematischen Logik, Math. Ann. 92 (1924) 305-316.

[24] J.R. Shoenfield, On degrees of unsolvability, Ann. of Math. 69 (1959) 644-653.

[25] C. Smoryński, Calculating self-referential statements, Fund. Math. 109 (1980) 189-210.

[26] R.I. Soare, Recursively Enumerable Sets and Degrees, Springer-Verlag, 1987.

[27] S.A. Terwijn, Generalizations of the recursion theorem, J. Symbolic Logic 83 (4) (2018) 1683-1690.

[28] S.A. Terwijn, The noneffectivity of Arslanov's completeness criterion and related theorems, arXiv:1804.01522, 2018.

[29] A. Turing, The p-function in $\lambda$ K-conversion, J. Symbolic Logic 2 (1937) 164.

[30] A. Visser, Numerations, $\lambda$-calculus, and arithmetic, in: J.P. Seldin, J.R. Hindley (Eds.), To H.B. Curry: Essays on Combinatory Logic, Lambda Calculus and Formalism, Academic Press, 1980, pp. 259-284. 